\title{
Methylprednisolone Treatment Versus Standard Supportive Care for Adult COVID-19 Mechanically Ventilated, Acute Respiratory Distress Syndrome Patients
}

\author{
Masood Ur Rahman ${ }^{1}$. Satish Chandrasekhar Nair ${ }^{2} \cdot$ Mehraj Ud Din $^{1} \cdot$ Mohammed Reidwan Dar ${ }^{1} \cdot$ Murriam Masood $^{3}$. \\ Al Reem Salem AI Menhali ${ }^{3}$. Mouza Mohammed AI Nuaimi ${ }^{3}$ Jayadevan Sreedharan ${ }^{4} \cdot$ Huda Imam Gasmelseed $^{4}$. \\ Asad Afroz Khan ${ }^{5}$
}

Accepted: 9 December 2021 / Published online: 3 January 2022

(c) The Author(s), under exclusive licence to Springer Nature Switzerland AG 2021

\begin{abstract}
A myriad of symptoms presented by severely ill mechanically ventilated COVID-19 patients has added pressure on the caregivers to explore therapeutic options. Systemic steroids have been reported to therapeutically benefit patients, with elevated inflammatory markers, during the severe acute respiratory syndrome, and the Middle East respiratory syndrome outbreak. COVID-19 disease is characterized by inflammation of the respiratory system and acute respiratory distress syndrome. Given the lack of specific treatment for COVID-19, the current study aimed to evaluate the therapeutic benefit of methylprednisolone as an add-on treatment for mechanically ventilated hospitalized COVID-19 patients with severe COVID pneumonia. Data were collected retrospectively from the electronic patient medical records, and interrater reliability was determined to limit selection bias. Descriptive and inferential statistical methods were used to analyze the data. The variables were crosstabulated with the clinical outcome, and the chi-square test was used to determine the association between the outcomes and other independent variables. Sixty-one percent (43/70) of the COVID-19 ARDS patients received standard supportive care, and the remainder were administered, methylprednisolone (minimum $40 \mathrm{mg}$ daily to a maximum $40 \mathrm{mg} \mathrm{q} 6 \mathrm{~h}$ ). A 28-day all-cause mortality rate, in the methylprednisolone group, was $18 \%(5 / 27, p<0.01)$ significantly lower, compared to the group receiving standard supportive care $(51 \%, 22 / 43)$. The median number of days, for the hospital length of stay (18 days), ICU length of stay ( 9.5 days), and the number of days intubated (6 days) for the methylprednisolone-treated group, was significantly lower $(p<0.01)$ when compared with the standard supportive care group. Methylprednisolone treatment also reduced the $\mathrm{C}$-reactive protein levels, compared to the standard care group on day 7. Our results strengthen the evidence for the role of steroids in reducing mortality, ICU length of stay, and ventilator days in mechanically ventilated COVID-19 patients with respiratory distress syndrome.
\end{abstract}

Keywords Acute respiratory distress syndrome $\cdot$ Gulf $\cdot$ Middle East $\cdot$ Steroids $\cdot$ Survival $\cdot$ Therapeutics

\section{Introduction}

The pandemic of a novel coronavirus-induced respiratory illness named coronavirus disease 2019 (COVID-19) has engulfed the world; the infectivity and the associated fatality have resulted in a global public health crisis and devastated

Masood Ur Rahman and Satish Chandrasekhar Nair contributed equally to the study

Satish Chandrasekhar Nair satchi2000@outlook.com

Extended author information available on the last page of the article economies [1]. The COVID-19 has infected millions, caused a significant fatality, and harmed the function of the immune system [2]. Acute respiratory distress syndrome (ARDS), cytokine storm, and elevated plasma levels of inflammatory cytokines correlate with disease severity, and poor prognosis [3, 4]. Given the lack of specific treatment for COVID-19, glucocorticoids and immunosuppressive treatment has been used to reduce the inflammation of the respiratory system, and prevent acute respiratory syndrome induction [4]. Methylprednisolone is a glucocorticoid used to suppress the autoimmune and inflammatory responses in rheumatic diseases and was administrated to patients during the earlier severe acute respiratory syndrome (SARS) and the Middle East 
respiratory syndrome outbreak $[5,6]$. A reduction in the all-cause 28-day mortality for COVID-19 patients, compared with standard care, was observed following the administration of systemic corticosteroids [7]. During the first wave of the contagion, between April 15 and June 15, 2020, the two public COVID-19 referral hospitals in the city of Al Ain, United Arab Emirates, adopted different protocols in the management of critically ill, COVID-19 patients. The Tawam hospital Intensive Care Unit (TICU) opted to treat patients with methylprednisolone. On the other hand, the $\mathrm{Al}$ Ain Hospital Intensive Care Unit (AICU) opted against steroid treatment and provided standard supportive care. The purpose of this study is to evaluate the therapeutic benefit of methylprednisolone as an add-on treatment in addition to standard supportive care for hospitalized COVID-19 patients with severe COVID pneumonia requiring mechanical ventilation. The therapeutic benefit was determined by the reduction in the 28-day all-cause mortality (primary outcome), and, the reduction in the number of days on mechanical ventilation, intensive care unit length of stay, hospital length of stay, and the levels of inflammatory markers, as secondary outcomes, in comparison to those patients that were provided standard supportive care.

\section{Methods}

\section{Study Design}

A cross-sectional retrospective observational study design of patients who tested positive for SARS-CoV-2 (COVID-19) was adopted [8].

\section{Setting}

Clinical data were collected from COVID-19 patients hospitalized at the only two public hospitals in $\mathrm{Al}$ Ain that catered to more than $98 \%$ of the total positive COVID-19 caseload. Largely, these two public hospitals address the secondary and tertiary care needs of the population of the eastern region of the United Arab Emirates. The study period was between April 15 and June 15, 2020, during the peak of the contagion in the UAE [9].

\section{Participants}

All the patients hospitalized for COVID-19 disease at the two public hospitals were selected for the study. The inclusion criteria involved hospitalized patients (a) 18 years of age or older, (b) all nationalities, (c) with a confirmed positive COVID-19 RT-PCR test result, (d) both male and female, (e) those initially seen at the emergency department, in-patient units, and the designated COVID-19 screening tents, (f) presenting with COVID-19 pneumonia and acute respiratory distress syndrome (ARDS) per the Berlin criteria [10], and (g) on mechanical ventilation. The exclusion criteria excluded patients (a) who died within $24 \mathrm{~h}$ of admission, (b) with a history of hypersensitivity to methylprednisolone, (c) diagnosed and living with human immunodeficiency virus or acquired immunodeficiency syndrome, (d) having a history of chronic use of corticosteroids, (e) diagnosed with decompensated cirrhosis, and/or chronic renal failure, (f) with heart failure as a predominant cause of acute respiratory failure, (g) undergone organ transplantation, (h) diagnosed with idiopathic pulmonary fibrosis, (i) with progressive neuromuscular disorders, (j) having dementia, (k) with decompensated psychiatric diseases, (1) on immunosuppressive treatments, (m) advised "Do Not Resuscitate" because of advanced age or morbid conditions, (n) below the age of 18 years (pediatric), and (o) with incomplete (demographic information) or missing (rapid antigen test instead of the RT-PCR for SARS-CoV-2) documentation. Following the assessment of patients for all-cause mortality on the $28^{\text {th }}$ day, the survivors were continued on supportive care, discharged, and/or sent to long-term care for recuperation.

\section{Variables}

The Al Ain Hospital Intensive Care Unit (AICU) adopted the Standard Supportive Care, protocol, where hospitalized patients received the ARDSnet protocol for ventilation, hydroxychloroquine along with standard intensive care unit (ICU) protocols for sedation, weaning, and glycemic control. In contrast, the patients at the Tawam Hospital Intensive Care Unit (TICU), in addition to standard supportive care, were administered methylprednisolone $(0.5 \mathrm{mg} / \mathrm{kg}$ per day, minimum dose $40 \mathrm{mg}$, and the maximum $40 \mathrm{q} 6 \mathrm{~h}$ ), continuously for a minimum of 5 days, and the maximum duration of treatment was left to the physician's discretion. The selection of the methylprednisolone dose was based on our clinical experience for patients with severe acute respiratory syndrome (SARS), a few years ago. The current study was conducted when there was no consensus on the standard dose of corticosteroid steroid usage for mechanically ventilated COVID-19 patients with ARDS before the results of the RECOVERY trial became available [11]. Serial measurements on days $0,1,3$, and 7 following methylprednisolone administration included ferritin, interleukin 6, C-reactive protein, D-dimer, and blood glucose levels, and compared with the patients on standard supportive care [12]. In addition, the date of admission, the 28-day all-cause mortality, the total number of days stayed in the hospital (hospital length of stay), the total number of days stayed in the intensive care unit, and the total number of days intubated were extracted from the electronic medical records of the hospital's validated patient information system. 


\section{Data Source}

The required clinical progress and patient outcomes data were abstracted from the patient electronic medical records of the Hospital Information System (Cerner, USA). Cerner system has been validated and used by all of the Abu Dhabi Health Services Company-managed public hospitals in the UAE since 2008 [13]. The data collected was recorded in the Microsoft Excel sheets, secured for authorized access by using a password. Standard statistical tests were applied to estimate the difference between the various variables, and are described under the "Statistical Methods" section.

\section{Bias}

The Steroid Tawam \& Alain Hospitals Research (STAR) group, consisting of physician-researchers, extracted data for the study from the patient medical records, but were blinded to the study hypothesis. Interrater reliability, Cohen's kappa coefficient, was used to assess the differences between the data abstractors [14]. Selection and operator bias were excluded by involving multiple data abstracters.

\section{Study Size}

The study period was during the peak of the contagion in the UAE; therefore, all the hospitalized adult COVID-19 patients, at both the public hospitals, were selected for the study. From a total of 83 patients, 70 patients that met the inclusion criteria were shortlisted and their data abstracted. The remainder of 16 patients was excluded from the study for the reasons listed under the "Participants" section.

\section{Statistical Methods}

Data were analyzed using SPSS (IBM, Chicago) Version 26. All hospitalized patients during the study period with COVID-19 were selected for the study, and shortlisted based on the inclusion criteria. Interrater reliability test was applied to assess the agreement of the data collected between the data abstractors. The Cohen kappa for the data abstractors was 0.72, indicating a good agreement. Descriptive and inferential statistical methods were used to analyze the data. First, the frequency and percentages are taken for all variables, and then the important variables are cross-tabulated with the outcome (deceased/survived). The chi-square test was used to find the association between the outcome variable and other independent variables. The level of significance was taken as $P<0.05$.

\section{Ethics Statement}

The methods of the study were carried out per the International Conference for Harmonization (ICH), and Good Clinical Practice guidelines. Waiver of written informed consents for participation in this retrospective observational study was granted by the Abu Dhabi Technical and Scientific Human Research Ethics Committee, a central research ethics committee at the department of health Abu Dhabi, UAE.

\section{Results}

A total of eighty-three patients between the two hospitals, confirmed positive for COVID-19 by RT-PCR, were shortlisted for the study. Elimination using the exclusion criteria resulted in a total of 70 patients. Sixty-one percent (43/70) of the COVID-19 ARDS patients were provided standard supportive care, per the standards of care protocol. Methylprednisolone was administered to thirty-nine percent of the COVID-19 ARDS patients (27/70), at a variable dose, $40 \mathrm{mg}$ daily to $40 \mathrm{mg} \mathrm{q} 6 \mathrm{~h}$ at the discretion of the intensivist, for a minimum of 5 days, consecutively. A 28-day all-cause mortality rate in the methylprednisoloneadministered group (TICU) was significantly $(p<0.01)$ lower, compared to the group (AICU) receiving standard supportive care. Almost eighty-two percent (22/27) of the COVID-19 ARDS patients treated with methylprednisolone survived (Table 1), as compared to 49\% (21/43) in the standard supportive care group. Striking differences in the hospital length of stay, intensive care unit length of stay, and the number of days intubated were also noted between the groups. The median number of days, for the hospital length of stay (18 days, 11-60, minimum-maximum), ICU length of stay (9.5 days, 5-34, minimum-maximum), and the number of days intubated (6 days, 2-22, minimum-maximum)
Table 1 Clinical outcomes of mechanically ventilated COVID-19 patients with ARDS, treated with methylprednisolone, in comparison to standard supportive care $(n=70)$

\begin{tabular}{|c|c|c|c|c|c|c|}
\hline \multirow{3}{*}{$\begin{array}{l}\text { Methylpredniso- } \\
\text { lone }\end{array}$} & \multicolumn{4}{|c|}{ Outcome } & \multirow[t]{3}{*}{ Total patients } & \multirow[t]{3}{*}{$P$ value } \\
\hline & \multicolumn{2}{|c|}{ Survived } & \multicolumn{2}{|c|}{ Deceased } & & \\
\hline & $n$ & $\%$ & $n$ & $\%$ & & \\
\hline Yes & 22 & 81.5 & 5 & 18.5 & 27 & $<0.01$ \\
\hline No & 21 & 48.8 & 22 & 51.2 & 43 & \\
\hline Total & 43 & 61.4 & 27 & 38.6 & 70 & \\
\hline
\end{tabular}


for the methylprednisolone-treated group, was significantly lower $(p<0.01)$ when compared with the standard supportive care group (Table 2). The blood glucose, ferritin, and D-dimer levels were not significantly different between the methylprednisolone-treated and the standard supportive care groups, on days 0 and 7 (data not shown). Almost all of the acute respiratory distress syndrome patients, irrespective of the assigned group, had high C-reactive protein levels on day 0. More than half (52\%) of methylprednisolone-treated ARDS COVID-19 patients showed normal levels of the C-reactive protein on day 7, compared to $19 \%$ for the standard supportive care group (data not shown).

\section{Discussion}

The spectrum of symptoms presented by the severely ill COVID-19 patients enhanced pressure on the caregivers to explore different therapeutic options [15]. A series of dysregulated host responses following inflammatory responses and subsequent organ injury has been reported to be the mechanism of severity for COVID-19 patients [16]. Patients with markedly elevated levels of the inflammatory markers are the potential candidates to benefit from systemic corticosteroids, as the therapeutic option to enhance anti-inflammation and immunosuppression [17]. Systemic corticosteroids that possess anti-cytokine activity, particularly methylprednisolone, are associated with good lung penetration [18-20]. Compared to other target-specific immunomodulating therapies, corticosteroids act broadly, inhibiting multiple inflammatory pathways [19]. The COVID-19 is associated with a cytokine profile depicted by activation of multiple inflammatory pathways involving interleukins, and other cytokines $[4,21]$. The methylprednisolone dose used in our study was higher when compared with the randomized control RECOVERY trial [11]. A significant reduction in the allcause 28-day mortality was observed in our study using methylprednisolone. Greater than $80 \%$ of the patients survived, compared to the subgroup with $48.8 \%$ survival for the patients on standard supportive care (Table 1), better than reported for the RECOVERY trial [11]. Recently, Go et al. [22] have provided conclusive evidence that a low dose of methylprednisolone decreases mortality in hospitalized COVID-19 patients on mechanical ventilation. The exact reason for the improvement in survival rate in our study is not known; partly, it may be attributed to the drug (methylprednisolone vs dexamethasone), the pragmatic dosing, the pharmacokinetic and pharmacodynamic advantages over dexamethasone, immunosuppressive effects, and the differential lung penetration [20]. In patients with severe COVID-19 pneumonia, early administration of prolonged, low-dose methylprednisolone treatment was associated with a significantly lower hazard of death and decreased ventilator dependence [15]. Methylprednisolone pulse administration at the beginning of the early pulmonary phase of illness has been reported to decrease the mortality rate and improve pulmonary involvement and oxygen saturation and downregulate inflammatory markers in COVID-19 patients [5]. Additionally, in our study, the mechanically ventilated COVID-19 patient with severe illness, treated with methylprednisolone, had shorter hospital length of stay, shorter ICU length of stay, and significantly reduced days of mechanical ventilation as compared with the standard supportive care group. No significant differences in the blood glucose level between the two groups were noted in our study. It has been reported by Wang et al. [23] that patients stressed by severe COVID-19 could produce abnormally high blood sugar levels, with no diabetes. A high level of fasting blood glucose $(\geq 7 \mathrm{mmol} / \mathrm{l})$ was found to be highly predictive of death and is independent of the severity of the patient's respiratory illness [23]. Neither, there was any significant increase in the overall adverse events between the groups. The C-reactive protein levels were lower in the methylprednisolone-treated group than the standard supportive care control group. The striking difference in the hospital length of stay between the two treatment groups can be explained by the fact that only mechanically ventilated patients were included in both groups. It is possible steroids are more effective in mechanically ventilated patients when compared to nonintubated patients with severe COVID-19 pneumonia [24].
Table 2 Improved secondary outcomes of mechanically ventilated COVID-19 patients with ARDS, treated with methylprednisolone, in comparison to standard supportive care $(n=70)$

\begin{tabular}{lllllll}
\hline $\begin{array}{l}\text { Methyl- } \\
\text { predniso- } \\
\text { lone }\end{array}$ & Primary outcome & Secondary outcome & Median (days) & Minimum & Maximum & $P$ value \\
\hline Yes & Survived & Hospital length of stay & 18 & 11 & 60 & $<0.01$ \\
No & & 39 & 14 & 77 & \\
Yes & Survived & ICU length of stay & 9.5 & 5 & 34 & $<0.01$ \\
No & & & 21 & 4 & 58 & \\
Yes & Survived & Days intubated & 6 & 2 & 22 & $<0.01$ \\
No & & & 12 & 1 & 27 & \\
\hline
\end{tabular}


Incidentally, the MetCovid trial [25] using methylprednisolone as an adjuvant $(0.5 \mathrm{mg} / \mathrm{kg}$ for 5 days) found no evidence of improved survival in the overall population in patients hospitalized for COVID-19. A delay in the start of methylprednisolone treatment, and the duration of treatment, may have been the potential factors that limited the survival of patients [25]. However, subgroup analysis for the same study indicated a lower mortality rate in patients treated with methylprednisolone, over 60 years old who presented a more pronounced systemic inflammatory status, as documented by high C-reactive protein values [25]. Uniquely, our study also included only COVID-19 patients on invasive ventilatory support following COVID pneumonia [26].

Our results are encouraging, especially in light of the fact that there are limited therapeutic interventions in the arsenal for COVID-19 management. Reduced mortality, lesser number of days on mechanical ventilation, and the shorter length of hospital and ICU stay reinforce the evidence in favor of using methylprednisolone in patients with severe COVID pneumonia with severe respiratory failure requiring mechanical ventilation. The retrospective nature of the study has limitations, such as data being static in the records, smaller sample size in each group, and potential inclusion biases. Data abstraction by multiple extractors has limited inclusion biases for the study.

In summary, despite these limitations, our findings strengthen the evidence for the role of steroids in reducing mortality, ICU length of stay, and ventilator days in mechanically ventilated COVID 19 patients with ARDS. Additionally, this is the first study in the Middle East region that addressed the therapeutic potential of methylprednisolone to reduce mortality; more importantly, the study was conducted during the peak of the first wave of the viral outbreak in the UAE. Further studies are needed to assess appropriate dosing and the superiority of methylprednisolone over dexamethasone in the clinical outcomes for mechanically ventilated COVID-19 patients with ARDS.

Acknowledgements The authors thank Tawam and Al Ain hospital management and the office of research governance at Tawam Hospital for their support to conduct the study.

Author Contribution Equal contribution (SN, MR); concept and design (SN); feasibility assessment (MR, AK); patient care (MR, AK, HQ, AM, MA, MM); literature search and updates ( $\mathrm{SN})$; data acquisition and organization (MD, MD, AA, MA); data analysis (JS), data interpretation (SN); manuscript preparation ( $\mathrm{SN}, \mathrm{MR}, \mathrm{AK})$; manuscript review and approval (all authors).

Data Availability The datasets generated and/or analyzed during the current study are not publicly available due to the institution's policy to code and archive data in a central repository of the hospital, but are available from the corresponding author on reasonable request.
Code Availability The data that support the findings of this study are available on reasonable request from the corresponding author.

\section{Declarations}

Ethics Approval The study was approved by Abu Dhabi COVID19 Research Ethics Committee. Approval Reference No. DOH/ CVDC/2020/1196.

Consent to Participate Waiver of written informed consents for participation in this retrospective observational study was granted by the Abu Dhabi Technical and Scientific Human Research Ethics Committee, a central research ethics committee at the department of health Abu Dhabi, UAE. No personal identifying information was collected for the patients; both subject privacy and patient data confidentiality were strictly adhered to in accordance with the international and national guidelines.

Consent for Publication Waiver of written informed consents for participation in this retrospective observational study was granted by the Abu Dhabi Technical and Scientific Human Research Ethics Committee, a central research ethics committee at the department of health Abu Dhabi, UAE. No personal identifying information was collected for the patients; both subject privacy and patient data confidentiality were strictly adhered to in accordance with the international and national guidelines.

Competing Interests The authors declare no competing interests.

\section{References}

1. Rothan HA, Byrareddy SN. The epidemiology and pathogenesis of coronavirus disease (COVID-19) outbreak. J Autoimmun. 2020;109: 102433. https://doi.org/10.1016/j.jaut.2020.102433.

2. Guan WJ, Ni ZY, Hu Y, Liang WH, Ou CQ, He JX, et al. China Medical Treatment Expert Group for COVID-19. Clinical characteristics of coronavirus disease 2019. China N Engl J Med. 2020;382(18):1708-20. https://doi.org/10.1056/NEJMoa2002032.

3. Iannaccone G, Scacciavillani R, Del Buono MG, Camilli M, Ronco C, Lavie CJ, et al. Weathering the cytokine storm in COVID-19: therapeutic implications. Cardiorenal Med. 2020;10(5):277-87. https://doi.org/10.1159/000509483.

4. Mehta P, McAuley DF, Brown M, Sanchez E, Tattersall RS, Manson JJ. HLH Across Speciality Collaboration, UK. COVID-19: consider cytokine storm syndromes and immunosuppression. Lancet. 2020;395(10229):1033-4. https://doi.org/10.1016/S01406736(20)30628-0.

5. Edalatifard M, Akhtari M, Salehi M, Naderi Z, Jamshidi A, Mostafaei $S$, et al. Intravenous methylprednisolone pulse as a treatment for hospitalised severe COVID-19 patients: results from a randomised controlled clinical trial. Eur Respir J. 2020;56(6):2002808. https://doi.org/10.1183/13993003. 02808-2020.

6. Kovarsky J. Intermediate-dose intramuscular methylprednisolone acetate in the treatment of rheumatic disease. Ann Rheum Dis. 1983;42(3):308-10. https://doi.org/10.1136/ard.42.3.308.

7. Sterne JAC, Murthy S, Diaz JV, Slutsky AS, Villar J, Angus DC, et al. Association between administration of systemic corticosteroids and mortality among critically ill patients with COVID-19: a meta-analysis. JAMA. 2020;324(13):1330-41. https://doi.org/10. 1001/jama.2020.17023. 
8. Abubakar AR, Sani IH, Godman B, Kumar S, Islam S, Jahan I, et al. Systematic review on the therapeutic options for COVID-19: clinical evidence of drug efficacy and implications. Infect Drug Resist. 2020;29(13):4673-95. https://doi.org/10.2147/IDR.S2890 37.

9. Sreedharan J, Nair SC, Muttappallymyalil J, Gopakumar A, Eapen NT, Satish KP, et al. Case fatality rates of COVID-19 across the globe: are the current draconian measures justified? Z Gesundh Wiss. 2021;24:1-9. https://doi.org/10.1007/s10389-021-01491-4.

10. Ranieri VM, Rubenfeld GD, Thompson BT, Ferguson ND, Caldwell E, Fan E, et al. Acute respiratory distress syndrome: the Berlin Definition. JAMA. 2012;307(23):2526-33. https://doi.org/10. 1001/jama.2012.5669.

11. Horby P, Lim WS, Emberson JR, Mafham M, Bell JL, Linsell L, et al. Dexamethasone in hospitalized patients with COVID-19. N Engl J Med. 2021;384(8):693-704. https://doi.org/10.1056/ NEJMoa2021436.

12. Boytsov SA, Pogosova NV, Paleev FN, Ezhov MV, Komarov AL, Pevsner DV, et al. Clinical characteristics and factors associated with poor outcomes in hospitalized patients with novel coronavirus infection COVID-19. Kardiologiia. 2021 Mar 1;61(2):4-14. Russian, English. https://doi.org/10.18087/cardio.2021.2.n1532.

13. Ibrahim H, Sorrell S, Nair SC, Al Romaithi A, Al Mazrouei S, Kamour A. Rapid development and utilization of a clinical intelligence dashboard for frontline clinicians to optimize critical resources during COVID-19. Acta Inform Med. 2020;28(3):20913. https://doi.org/10.5455/aim.2020.28.209-213.

14. McHugh ML. Interrater reliability: the kappa statistic. Biochem Med (Zagreb). 2012;22(3):276-82.

15. Cano EJ, Fonseca Fuentes X, CorsiniCampioli C, O'Horo JC, Abu Saleh O, Odeyemi Y, et al. Impact of corticosteroids in coronavirus disease 2019 outcomes: systematic review and meta-analysis. Chest. 2021;159(3):1019-40. https://doi.org/10.1016/j.chest.2020. 10.054 .

16. Mudd PA, Remy KE. Prolonged adaptive immune activation in COVID-19: implications for maintenance of long-term immunity? J Clin Invest. 2021;131(1): e143928. https://doi.org/10.1172/ JCI143928.

17. Figliozzi S, Masci PG, Ahmadi N, Tondi L, Koutli E, Aimo A, et al. Predictors of adverse prognosis in COVID-19: a systematic review and meta-analysis. Eur J Clin Invest. 2020;50(10): e13362. https://doi.org/10.1111/eci.13362.

18. Arabi YM, Mandourah Y, Al-Hameed F, Sindi AA, Almekhlafi GA, Hussein MA, et al. Corticosteroid therapy for critically ill patients with Middle East respiratory syndrome. Am J Respir Crit Care Med. 2018;197(6):757-67. https://doi.org/10.1164/rccm. 201706-1172OC.

19 Salton F, Confalonieri P, Meduri GU, Santus P, Harari S, Scala $\mathrm{R}$, et al. Prolonged low-dose methylprednisolone in patients with severe COVID-19 pneumonia. Open Forum Infect Dis. 2020;7(10):ofaa421. https://doi.org/10.1093/ofid/ofaa421.

20. Braude AC, Rebuck AS. Prednisone and methylprednisolone disposition in the lung. Lancet. 1983;2(8357):995-7.

21. Xu Z, Shi L, Wang Y, Zhang J, Huang L, Zhang C, et al. Pathological findings of COVID-19 associated with acute respiratory distress syndrome. Lancet Respir Med. 2020;8(4):420-2. https:// doi.org/10.1016/S2213-2600(20)30076-X.

22. Go RC, Shah R, Ntirenda T, Oe Y, Sarfraz K, et al. Methylprednisolone and 60 days in hospital survival in coronavirus disease 2019 pneumonia. Crit Care Explor. 2021;3(7): e0493. https://doi. org/10.1097/CCE.0000000000000493.

23. Wang S, Ma P, Zhang S, Song S, Wang Z, et al. Fasting blood glucose at admission is an independent predictor for 28-day mortality in patients with COVID-19 without previous diagnosis of diabetes: a multi-centre retrospective study. Diabetologia. 2020;63(10):2102-11. https://doi.org/10.1007/ s00125-020-05209-1.

24. Papamanoli A, Yoo J, Grewal P, Predun W, Hotelling J, Jacob $\mathrm{R}$, et al. High-dose methylprednisolone in nonintubated patients with severe COVID-19 pneumonia. Eur J Clin Invest. 2021;51(2): e13458. https://doi.org/10.1111/eci.13458.

25. Jeronimo CMP, Farias MEL, Val FFA, Sampaio VS, Alexandre MAA, Melo GC, et al. Methylprednisolone as adjunctive therapy for patients hospitalized with coronavirus disease 2019 (COVID19; Metcovid): a randomized, double-blind, phase IIb, placebocontrolled trial. Clin Infect Dis. 2021;72(9):e373-81. https://doi. org/10.1093/cid/ciaa1177.

26 Fadel R, Morrison AR, Vahia A, Smith ZR, Chaudhry Z, Bhargava P, et al. Henry Ford COVID-19 Management Task Force. Early short-course corticosteroids in hospitalized patients with COVID-19. Clin Infect Dis. 2020;71(16):2114-20. https://doi.org/ 10.1093/cid/ciaa601.

Publisher's Note Springer Nature remains neutral with regard to jurisdictional claims in published maps and institutional affiliations. 


\section{Authors and Affiliations}

Masood Ur Rahman ${ }^{1}$. Satish Chandrasekhar Nair ${ }^{2} \cdot$ Mehraj Ud Din ${ }^{1} \cdot$ Mohammed Reidwan Dar $^{1} \cdot$ Murriam Masood $^{3}$. Al Reem Salem Al Menhali ${ }^{3}$. Mouza Mohammed Al Nuaimi ${ }^{3}$ Jayadevan Sreedharan ${ }^{4} \cdot$ Huda Imam Gasmelseed $^{4}$. Asad Afroz Khan ${ }^{5}$

\author{
Masood Ur Rahman \\ mrahman@seha.ae \\ Mehraj Ud Din \\ mbhat@seha.ae \\ Murriam Masood \\ o-mmasood@seha.ae \\ Jayadevan Sreedharan \\ drjayadevans@gmail.com \\ Huda Imam Gasmelseed \\ drhudah@hotmail.com \\ Asad Afroz Khan \\ Akhan@seha.ae
}

1 Department of Critical Care Medicine, Tawam Hospital, Al Ain, United Arab Emirates

2 Department of Academic Affairs, Tawam Hospital, College of Medicine, UAE University, Al Ain, United Arab Emirates

3 Department of Academic Affairs, Internal Medicine Residency Program, Tawam Hospital, Al Ain, United Arab Emirates

4 Department of Community Medicine, Gulf Medical University, Ajman, United Arab Emirates

5 Department of Infectious Diseases, Tawam Hospital, Al Ain, United Arab Emirates 